\title{
Sequential treatment of metastatic renal cancer in a complex evolving landscape
}

\author{
Javier C. Angulo ${ }^{1}$, Charles H. Lawrie ${ }^{2}$, José I. López ${ }^{3}$ \\ ${ }^{1}$ Clinical Department, Faculty of Biomedical Sciences, European University of Madrid, Getafe, Madrid, Spain; ${ }^{2}$ Molecular Oncology, Biodonostia \\ Research Institute, San Sebastián, Spain; ${ }^{3}$ Department of Pathology, Cruces University Hospital, Biocruces-Bizkaia Research Institute, University of \\ the Basque Country (UPV/EHU), Leioa, Barakaldo, Spain \\ Correspondence to: Dr. José I. López. Department of Pathology, Cruces University Hospital, Plaza de Cruces s/n, 48903 Barakaldo, Spain. \\ Email: jilpath@gmail.com. \\ Provenance: This is an invited article commissioned by the Section Editor Dr. Xiao Li (Department of Urology, Jiangsu Cancer Hospital, Jiangsu \\ Institute of Cancer Research, Nanjing Medical University Affiliated Cancer Hospital, Nanjing, China). \\ Comment on: Voog E, Campillo-Gimenez B, Elkouri C, et al. Long survival of patients with metastatic clear cell renal cell carcinoma. Results of real \\ life study of 344 patients. Int J Cancer 2019. [Epub ahead of print].
}

Submitted Oct 08, 2019. Accepted for publication Nov 29, 2019.

doi: $10.21037 / \mathrm{atm} .2019 .12 .05$

View this article at: http://dx.doi.org/10.21037/atm.2019.12.05

The large armamentarium of therapies available today for metastatic clear cell renal cell carcinoma (mCCRCC) has prolonged the survival of these patients in real-life as many of them are treated outside clinical trials with a sequential treatment strategy. The paper recently published by Voog et al. (1) confirms the observation most clinicians share that a large proportion of patients with good or intermediate prognosis who progress after two lines of treatment still have a performance status acceptable enough to furtherly receive additional systemic therapy, thus conferring this dreadful disease a longer survival expectancy. The overall survival they report was 57 months in patients with good or intermediate prognosis and 19 months in patients with bad prognosis, classified according to the International Metastatic Renal Cell Carcinoma Database Consortium (IMDC).

However, the clinician treating a patient with mCCRCC currently faces the difficult task to choose the most appropriate therapeutic regimen in a rapidly developing field with recommendations mainly derived from clinical trials, because patient characteristics and survival outcomes in randomized trials may be different from those in reallife clinical practice. On the one hand, clinical assessment in real world practice to determine progression are not as frequent as in clinical trials and, on the other, general patient population treated in routine clinical practice is more heterogeneous and likely to be elderly and/or less healthy. Additionally, the choice of first and second therapy has changed a lot in the last two decades, but not that much from 2011 to 2014, the time of recruitment for the IVORE (Étude observationnelle prospective évaluant les traitments par Voie Orale contre le cancer métastatique du Rein) cohort published by Voog et al. (1) using sequential treatments. Tyrosine kinases inhibitors (TKI) sunitinib, pazopanib or sorafenib were used as first-line treatment in $91.7 \%$ of the patients. Predominantly the second line choice was the inhibitor of the mammalian target of rapamycin pathway (imTOR) everolimus in $53.3 \%$ of cases, but other options were the same TKI used as first-line in $23.1 \%$, and the recently approved TKI axitinib in $22.7 \%$ of cases. For third-line therapy $63.5 \%$ of patients received TKI, primarily sorafenib and axitinib (23.5\% each), whereas $36.5 \%$ of patients received everolimus. For fourth-line and beyond, sorafenib was used in $21.2 \%$ of patients as fourth-line therapy, and both sunitinib and everolimus was used equally as fifth-line therapy, in $23.8 \%$ of patients. These figures reflect the real scenario of the first half of this decade, as axitinib after prior sunitinib (2), pazopanib followed by everolimus (3), or sunitinib rechallenge after other targeted therapies (4) have vied to define the optimal therapy for patients with mCCRCC whose disease progresses after initial vascular endothelial growth factor receptor-tyrosine kinase inhibitor (VEGFR-TKI) treatment.

Voog et al. (1) prospectively confirm that treatment with 
a second or third TKI provides additional clinical benefit without cumulative toxicity in unselected patients, and also that rechallenge, defined as using a treatment class that previously produced duration of tumor control over 6 months in patients with good or intermediate prognosis according to the IMDC, is feasible. However, it should be born in mind that the clinical scenario they present has changed greatly over the last couple of years with the advent of the multi-kinase inhibitor cabozantinib, and the introduction of immunotherapy with monoclonal anti-bodies nivolumab, ipilimumab, pembrolizumab, atezolizumab and avelumab, that target the immune checkpoint proteins PD-1, PD-L1 and CTLA-4. Equally important to consider is that the introduction of these treatments mean that there are now many more alternatives for the clinician to choose between when treating mCCRCC, and that in the absence of clear guidelines it is much more difficult to decide or indeed analyse which is the most effective regimen in the way that was possible for the period covered by the IVORE study. Additionally, the IMDC risk categories have shifted based on the results of the Checkmate-214 and Keynote-426 trials $(5,6)$, and now tend to be defined as "good" (favourable) and "bad" (intermediate/poor), as immune checkpoint inhibition (ICI) is now the first-line treatment for mCCRCC as promoted by international consensus (7). The survival benefit of a double checkpoint blockade combination, ipilimumab and nivolumab, when compared to sunitinib in intermediate and poor risk mCCRCC is now clear $(5,8)$. The combination pembrolizumab plus axitinib has also been recommended, not only for unfavorable disease but also for patients who fit the favorable risk category (6). These achievements place us closer to an individualized patient therapy for mCCRCC $(9,10)$. In the near future, robust data consolidation balancing efficacy, safety and quality of life will give valuable information on the role of VEGFR-TKI plus ICI combination. Several clinical trials are currently underway that are testing other combinations including pazopanib plus nivolumab (NCT01472081), pazopanib plus pembrolizumab (NCT02014636), axitinib plus avelumab (Javelin Renal-101), axitinib plus pembrolizumab (NCT02853331), lenvatinib plus pembrolizumab (NCT02811861), cabozantinib plus nivolumab (NCT03141177), cabozantinib plus pembrolizumab (NCT03149822), tivozanib plus nivolumab (NCT03136627) and cabozantinib plus nivolumab plus ipilimumab (NCT02496208) $(8,10)$. In the meantime, VEGFR-TKI sunitinib and pazopanib are recommended as alternative first-line therapeutic regimens for patients who cannot tolerate ICI, and cabozantinib remains a valid substitute option for the intermediate and high-risk group within a first-line setting $(7,9)$.

Nobody doubts that with the introduction of ICI and next-generation VEGFR-TKI the survival of patients with advanced renal cell cancer will improve remarkably once synergistic combinations in the process of development expand the therapeutic armamentarium of first-line and rescue therapies. Fortunately, the number of prior therapies does not seem to affect the efficacy of cabozantinib or nivolumab (8). Such therapeutic options may facilitate long-term disease control in a substantial proportion of patients thereby improving the current situation with VEGFR-TKI alone (11). That said it remains uncertain whether a population of patients with mCCRCC could be described to be cured indefinitely. Therefore, the need for an optimum strategy for treatment sequencing after failure of immunotherapy remains a mystery and surely future treatment strategies should focus, not only on efficacy but also on safety and quality of life aspects. However, the rapidness of evolving therapies and standards in mCCRCC also frustrates studies to evaluate treatment sequencing, as they may become obsolete during the study life. Studies regarding switching from a targeted therapy to ICI and vice versa will give very valuable information upon how effective sequencing should be accomplished. In the meantime, the optimum duration of PD-1/PD-L1 inhibitors use and the length of response remain unclear. Additionally, progression free survival (PFS) may not be the best parameter to evaluate efficacy of ICI therapy as a tumor reduction after progression with nivolumab maintenance has been described $(8,11)$.

For patients progressing with prior VEGF targeted therapy, nivolumab, cabozantinib, axitinib or the combination of ipilimumab and nivolumab appear to be the most plausible alternatives. However, the main dilemma to sequence treatments would be second-line treatment for patients previously treated with ICI. Most likely any VEGFR-TKI not previously used in combination with ICI therapy is a valid option $(7,9,12)$. Also, keeping in mind the strategy to reserve some VEGFR-TKI options (cabozantinib) or mTOR including combinations (lenvatinib plus everolimus) for a later rescue line seems advisable, but no serious evidence is available at this point to back this stance (8). Another interesting question will be the possibility of ICI rechallange, and elucidating the use of cytoreductive nephrectomy within the context of new 
immunotherapeutic interventions. Better markers of response to ICI need to be identified before the optimal selection of therapy can be determined for the individual patient. This goes in hand with strategies for a better molecular characterization in the individual patient taking also into account the well admitted heterogeneity of renal cancer. Probably the concept of "lines of therapy" to treat mCCRCC as traditionally considered by clinicians should give way to a real personalized medicine to elect among the many available therapies those with maximal chance of long-term response and minimal risk of adverse effects for a precise patient.

Undoubtfully, future development of biomarkers predictive of response will be critical to optimise treatment individualization. Tumor microenvironment dynamics in mCCRCC characterizing angiogenesis and inflammatory signatures may help to define prediction of response to target therapy using VEGR-TKI and/or ICI (13). The integration of stromal and immune biomarkers should be evaluated regarding the inherent heterogeneity of this disease. Tumor associated macrophages (TAMs) play an important role in both VEGFR-TKI and ICI therapy resistance and multiregion assessment using high-resolution technologies like single-cell RNA-seq could clarify tumor microenvironment and its relationship with immunotherapy outcome (13-15).

Despite the remarkable survival benefit obtained by some CCRCC patient populations to ICI based therapy, around $40-60 \%$ of patients do not respond, resulting in unnecessary costs and associated toxicities. There is therefore a clear necessity for improving predictive ICI biomarkers used in the clinic to better select responsive patients. Detection of PD-L1 by immunohistochemistry is by far the most commonly used predictive biomarker for ICI therapy. PDL1 is expressed in 14-66\% of CCRCC cases, depending on the study design, either in tumour cells or tumor-infiltrating lymphocytes (TILs). Although, several studies demonstrate that PD-L1 positive CCRCC tumours achieve a better response to ICI therapies (16), its use remains controversial and between $8-17 \%$ of PD-L1 negative patients also responded to treatment (17). Indeed, in the checkmate 025 trial, that formed the basis of FDA-approval of nivolumab in ccRCC, the survival benefit was found to be independent of PD-L1 status (18). The usefulness of PD-L1expression is further compromised by the use of differing companion antibodies with different cut-offs and scoring systems and that have low levels of reproducibility between the 22-C3 and 28-8 antibodies, and lower levels of SP142 sensitivity for PD-L1 expression in tumour cells (19). Furthermore, PD-L1 expression is dynamic over the course of the tumour progression and is modified by antiangiogenic therapy and is also differentially expressed in different parts of the tumour [i.e., intratumor heterogeneity (ITH) see below] (20). Several alternative biomarkers have been proposed including gene expression signatures and tumour mutational burden (TMB), defined as the number of mutations per coding area of the tumour genome. However, these alternatives are yet to be rigorously tested and are beyond the means of many hospitals. As a consequence, it is likely that the improvements brought about by ICI therapy for CCRCC patients remain to be fully realized without a more specific biomarker, and reinforce the need for real-life studies as opposed to relying on solely clinical trials to set procedures as implied by the paper of Voog et al. (1) within the TKI setting. It should also be borne in mind that the use of companion diagnostic biomarkers is an increasingly frequent requirement of regulatory approval for new therapeutics, and so can represent a compromise situation by the pharma industry even when the probable (and lucrative) outcome is that the weight of evidence suggest that all CCRCC patients are treated irrespective of biomarker status.

Very recent molecular studies have shown the genomic complexity of CCRCC. Spatial and temporal evolution develop CCRCC tumor regions with different molecular signatures. This ITH has been classically considered a pure stochastic process. However, Turajlic et al. (14) have found up to seven deterministic evolutionary pathways in this tumor with direct impact in tumor evolution and clinical aggressiveness. Some genomic profiles have been linked to aggressive clinical behavior, including cases with BAP-1 driven mutations, cases with mutations in multiple clonal drivers, and cases with VHL wild-type (14). Other genomic signatures, however, confer less aggressive behaviors and longer survival rates to patients, for example $\mathrm{PBRM} 1 \rightarrow \mathrm{SETD} 2, \mathrm{PBRM} 1 \rightarrow \mathrm{PI} 3 \mathrm{~K}, \mathrm{PBRM} 1 \rightarrow \mathrm{SCNA}$ driven mutations and VHL mono-driven mutation (14).

The metastatic competence in CCRCC is afforded by chromosome complexity, as stated by Turajlic et al. in a recent study of 101 cases (15), 9p and 14q losses being the genomic hallmark detected in the metastases. Interestingly, the seven previously described deterministic evolutionary patterns in the primary tumors correlate with specific patterns of metastases (15). This way, the aggressive genotypes develop multiple and early metastases whereas VHL mono-driven cases, for example, never metastasize. 
Since molecular signatures of biological aggressiveness do not always correlate with histological high-grade areas, and since high grade areas are not always detected by naked eye during tumor sampling, the problem of the reliability of representativeness of most tumors is a crucial unresolved issue. An additional problem dealing mCCRCC is that tumor sample from minimally invasive biopsy is always scarce when cytoreductive nephrectomy is not performed, a hot issue that is even more controversial with the advent of immunotherapy.

Precision therapy needs precision pathology first to unveil the complex molecular landmark of a significant number of CCRCC. Recent studies have shown that current protocols for tumor selection are insufficient to detect ITH with reliability $(21,22)$. On the other hand, a total tumor sampling, although optimal, is unsustainable due to the big size of many CCRCC at the time of diagnosis. Therefore, the main question is: how extensive a tumor sampling must be? or in other words, when to stop sampling? An attempt to trade off costs and benefits has been published recently: the multisite tumor sampling $(21,22)$. This method proposes a random sampling of many regions within the same tumor trimming it in small fragments able to be included in a few paraffin blocks keeping this way the cost affordable.

Since tumor sampling is still an unresolved problem with direct impact in patients, alternative attempts have appeared recently as promising tools trying to help resolving this issue. A study using 3D-printed molds of renal tumors for image-guided tissue sampling has been deposited in Biorxiv very recently (23). Likewise, mathematics could be useful for such purpose, for instance, a game theory approach is being lately proposed as a promising tool to extract hidden information from data series of treatment response and clinical evolution of breast (24) and prostate (25) tumors.

Unfortunately, we must admit that despite the wide therapeutic offer available current clinical-pathological knowledge is still very far from being able to define personalized therapeutic approach for a patient and, consequently, optimal sequential therapies to be adopted in the particular case.

\section{Acknowledgments}

None.

\section{Footnote}

Conflicts of Interest: The authors have no conflicts of interest to declare.

Ethical Statement: The authors are accountable for all aspects of the work in ensuring that questions related to the accuracy or integrity of any part of the work are appropriately investigated and resolved.

\section{References}

1. Voog E, Campillo-Gimenez B, Elkouri C, et al. Long survival of patients with metastatic clear cell renal cell carcinoma. Results of real life study of 344 patients. Int J Cancer 2019. [Epub ahead of print].

2. Kadono Y, Konaka H, Izumi K, et al. Efficacy and safety of cytokines versus first-line sunitinib and second-line axitinib for patients with metastatic renal cell carcinoma (ESCAPE study): A study protocol for phase III randomized sequential open-label study. Contemp Clin Trials Commun 2019;15:100403.

3. Rossetti S, D'Aniello C, Iovane G, et al. Sequential treatment with pazopanib and everolimus in metastatic renal cell carcinoma. Front Pharmacol 2017;8:484.

4. Munárriz J, Reynés G, Sánchez-Lorenzo L, et al. Sunitinib rechallenge in advanced renal cell carcinoma: outcomes of a multicenter retrospective study. Cancer Chemother Pharmacol 2019;84:781-9.

5. Motzer RJ, Tannir NM, McDermott DF, et al. Nivolumab plus ipilimumab versus sunitinib in advanced renal-cell carcinoma. N Engl J Med 2018;378:1277-90.

6. Rini BI, Plimack ER, Stus V, et al. Pembrolizumab plus Axitinib versus sunitinib for advanced renal-cell carcinoma. N Engl J Med 2019;380:1116-27.

7. Albiges L, Powles T, Staehler M, et al. Updated European Association of Urology guidelines on renal cell carcinoma: Immune checkpoint inhibition is the new backbone in first-line treatment of metastatic clear-cell renal cell carcinoma. Eur Urol 2019;76:151-6.

8. de Velasco G, Bex A, Albiges L, et al. Sequencing and combination of systemic therapy in metastatic renal cell carcinoma. Eur Urol Oncol 2019;2:505-14.

9. Angulo JC, Shapiro O. The Changing Therapeutic landscape of metastatic renal cancer. Cancers (Basel) 2019;11. doi: 10.3390/cancers11091227.

10. Kotecha RR, Motzer RJ, Voss MH. Towards individualized therapy for metastatic renal cell carcinoma. Nat Rev Clin Oncol 2019;16:621-33.

11. Lakomy R, Poprach A, Bortlicek Z, et al. Utilization and efficacy of second-line targeted therapy in metastatic renal 
cell carcinoma: data from a national registry. BMC Cancer 2017;17:880.

12. Shah AY, Kotecha RR, Lemke EA, et al. Outcomes of patients with metastatic clear-cell renal cell carcinoma treated with second-line VEGFR-TKI after firstline immune checkpoint inhibitors. Eur J Cancer 2019;114:67-75.

13. Vuong L, Kotecha RR, Voss MH, et al. Tumor microenvironment dynamics in clear-cell Renal cell carcinoma. Cancer Discov 2019;9:1349-57.

14. Turajlic S, Xu H, Litchfield K, et al. Deterministic evolutionary trajectories influence primary tumor rrowth: TRACERx Renal. Cell 2018;173:595-610.e11.

15. Turajlic S, Xu H, Litchfield K, et al. Tracking cancer evolution reveals constrained routes to metastases: TRACERx Renal. Cell 2018;173:581-594.e12

16. Topalian SL, Hodi FS, Brahmer JS, et al. Safety, activity, and immune correlates of anti-PD-1 antibody in cancer. $\mathrm{N}$ Engl J Med 2012;366:2443-54.

17. Khagi Y, Kurzrock SP, Patel SP. Next generation predictive biomarkers for immune checkpoint inhibition. Cancer Metastasis Rev 2017;36:179-90.

18. Motzer RJ, Escudier B, McDermott DF, et al. Nivolumab versus everolimus in advanced renal-cell carcinoma. $\mathrm{N}$ Engl J Med 2015;373:1803-13.

19. Tsao MS, Kerr KM, Kockx M, et al. PD-L1

Cite this article as: Angulo JC, Lawrie CH, López JI. Sequential treatment of metastatic renal cancer in a complex evolving landscape. Ann Transl Med 2019;7(Suppl 8):S272. doi: 10.21037/atm.2019.12.05 immunohistochemistry comparability study in real-life clinical samples: Results of Blueprint Phase 2 Project. J Thorac Oncol 2018;13:1302-11.

20. López JI, Pulido R, Cortés JM, et al. Potential impact of PD-L1 (SP-142) immunohistochemical heterogeneity in clear cell renal cell carcinoma immunotherapy. Pathol Res Pract 2018;214:1110-4.

21. López JI, Cortés JM. A divide-and-conquer strategy in tumor sampling enhances detection of intratumor heterogeneity in pathology routine: A modeling approach in clear cell renal cell carcinoma. F1000Res 2016;5:385.

22. López JI, Cortés JM. Multi-Site Tumor Sampling (MSTS): A new tumor selection method to enhance intratumor heterogeneity detection. Hum Pathol 2017;64:1-6.

23. Crispin-Ortuzar M, Gehrung M, Ursprung S, et al. 3D-printed moulds of renal tumours for image-guided tissue sampling in the clinical setting. BioRxiv 2019. doi. org/10.1101/658831.

24. West J, Robertson-Tessi M, Luddy K, et al. The immune checkpoint kick start: Optimization of neoadjuvant combination therapy using game theory. JCO Clin Cancer Inform 2019;3:1-12.

25. Zhang J, Cunningham JJ, Brown JS, et al. Integrating evolutionary dynamics into treatment of metastatic castrate-resistant prostate cancer. Nat Commun 2017;8:1816. 